\title{
Commissioning simulations for the Argonne Advanced Photon Source upgrade lattice
}

\author{
V. Sajaev* \\ Advanced Photon Source, Argonne National Laboratory, Argonne, Illinois 60439, USA
}

(Received 18 September 2018; published 26 April 2019)

\begin{abstract}
A hybrid seven-bend-achromat lattice that features very strong focusing elements and a relatively small vacuum chamber has been proposed for the Advanced Photon Source (APS) upgrade. The design lattice parameters during commissioning will need to be quickly achieved in order to minimize the interruption of user operation. This paper describes start-to-end simulation of the machine commissioning beginning from first-turn trajectory correction, progressing to orbit correction, and culminating in lattice correction and coupling adjustment. The automated commissioning procedure shows that the rapid commissioning of the ultralow emittance lattice is possible. It also enables a more rigorous statistical evaluation of expected performance of proposed lattices.
\end{abstract}

DOI: 10.1103/PhysRevAccelBeams.22.040102

\section{INTRODUCTION}

The Advanced Photon Source [1] is a 7-GeV, 100-mA, 40 -sector 3rd generation storage ring light source with a $1104-\mathrm{m}$ circumference, providing beams to dozens of insertion device (ID) and bending magnet (BM) beamlines simultaneously. After more than 20 years of operation, a major upgrade of the lattice is under consideration.

APS has a large user community who insist that facility "dark time" (the time when APS is not providing synchrotron light to users) during the upgrade is minimized. To satisfy this requirement, APS is targeting 12 months for removal of old magnets, installation of new ones, and commissioning. Of this 12 month period, only three months are set aside for commissioning of the new multibend achromat ring.

Several lattices were under consideration for the APS upgrade. All were based on the hybrid 7-bend achromat approach first introduced at ESRF [2]. The lattices have natural emittances that range from 40 to $90 \mathrm{pm}$, which is 70 to 30 times smaller than the present APS ring. Figure 1 shows lattice functions for the 41-pm lattice, which is presently chosen as the preliminary design for the project. The lattice properties are listed in Table I; other lattice details are described in [3].

The ultralow emittance of this lattice is achieved by much stronger focusing than in the present ring. For

\footnotetext{
*sajaev@aps.anl.gov
}

Published by the American Physical Society under the terms of the Creative Commons Attribution 4.0 International license. Further distribution of this work must maintain attribution to the author(s) and the published article's title, journal citation, and DOI. example, maximum quadrupole strengths increase nearly five-fold in the new lattice compared to the present APS lattice. Stronger focusing inevitably leads to larger natural chromaticity and thus a nearly seven-fold increase in sextupole strength is needed, resulting in rather small dynamic aperture and short lifetime even for the ideal lattice. Misalignments of the strong quadrupoles generate large orbit errors, which in the presence of very strong sextupoles leads to huge lattice and coupling errors. Another difficulty originates in the smaller vacuum chamber apertures that are required to achieve high gradients in the magnets. In addition, in 3rd-generation light sources the small-gap ID chambers are usually installed after commissioning, in order to make commissioning easier. In the APS upgrade, almost all of these chambers-which are generally smaller than those in present rings-will be installed prior to commissioning, in order to facilitate moving directly into operation once commissioning is completed. Another significant challenge arises from limited orbit corrector strengths. Due to compactness of the lattice, the correctors in the new machine will be five to ten times weaker than in the present APS while the quadrupole kicks due to misalignments will be five times stronger. These many factors suggest that the hoped-for three-month commissioning period will be very challenging. This paper addresses this question using a realistic simulation of the commissioning process.

Rapid commissioning is important for any facility, and most modern accelerators were commissioned fairly quickly. A brief survey of recently commissioned synchrotron light sources can be found in [4], where it is stressed that the keys to commissioning success include thorough subsystem commissioning without beam and having controls software tested ahead of time. An additional case 


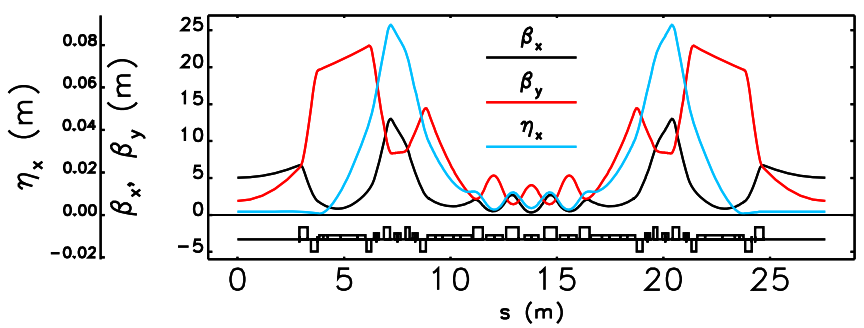

FIG. 1. Lattice functions for the APS upgrade lattice: horizontal beta function (black), vertical beta function (red), and horizontal dispersion function (blue).

study is LHC commissioning, for which detailed procedures for measurement and correction of optics were developed via simulations and measurements at other facilities [5]. Thanks to this preparation, the optics measurement and correction software was developed in advance [6], and the optics measurement and correction at LHC was carried out immediately after stored beam was achieved $[7,8]$. NSLS-II experience [9] provides another example of rapid commissioning, and included use of a simulator [10], again for software development rather than simulation of commissioning. These experiences of rapid commissioning thanks to early application of correction algorithms reinforces our belief that the effort spent on developing the commissioning simulation software will pay off during commissioning of the APS upgrade lattice.

Another important topic with highly nonlinear lattices is the proper evaluation of lattice performance in the presence of magnet and alignment errors. It is well known that errors degrade the performance of the machine by breaking symmetry and exciting nonsystematic resonances. In order to evaluate the lattice in the presence of errors, one needs to introduce some errors in simulation. However, introducing realistic errors would lead to very large orbit and lattice distortions, because real machines never operate without correction to reduce these errors. Essentially, one cannot generate lattices with realistic errors without going through some sort of commissioning process. The shortest way to generate lattices with realistic errors for performance evaluation includes these steps: generation of error sets with realistic magnet strength and alignment errors, correction of the closed orbit, and then correction of the optics. Example of such evaluation can be found in the SLS-2 Conceptual Design Report [11]. This approach works better for smaller machines, but for larger machines like APS it becomes hard to use, because the closed orbit does not exist for large percentage of error sets (more details on this will be given later). In this case, one has to start from the first-turn trajectory correction and actually go through the entire commissioning process similar to the commissioning of the real machine. Commissioning simulations presented here do exactly that, and allow one to generate commissioned lattices for performance evaluation [3].

TABLE I. Lattice parameters.

\begin{tabular}{lcc}
\hline \hline Beam energy & 6 & $\mathrm{GeV}$ \\
\hline$\nu_{x, y}$ & $95.12,36.12$ & \\
Natural chromaticity $\xi_{x, y}$ & $-139,-108$ & \\
Maximum $\beta_{x, y}$ & $12.9,18.9$ & $\mathrm{~m}$ \\
Maximum dispersion $\eta_{x}$ & 0.074 & $\mathrm{~m}$ \\
Natural emittance & 41 & $\mathrm{pm}$ \\
Energy spread & 0.13 & $\%$ \\
x,y,z damping time & $7.2,15.8,19.9$ & $\mathrm{~ms}$ \\
Energy loss per turn & 2.80 & $\mathrm{MeV}$ \\
Momentum compaction & $3.7 \times 10^{-5}$ & \\
Circumference & 1103.98 & \\
$\beta_{x, y} @$ IDs & $5.0,1.9$ & $\mathrm{~m}$ \\
$\eta_{x} @$ IDs & 1.5 & $\mathrm{~mm}$ \\
Effective emittance @ IDs & 41.0 & $\mathrm{pm}$ \\
\hline \hline
\end{tabular}

\section{EXPECTED PERFORMANCE OF THE UNCORRECTED LATTICE}

As mentioned above, it is clear that without applying any orbit and lattice correction, one should expect large orbit and beta function errors when first injecting into the ring. To understand the scale of difficulties that awaits during commissioning of a machine like APS-U, one can simply try to calculate orbit and lattice errors with the expected magnet strength and alignment errors without applying any correction. Table II shows the expected magnet strength and alignment errors that were used in simulations. To obtain the dependence of the uncorrected lattice performance on the magnitude of magnet errors, the error values were scanned as a fraction of the numbers given in Table II. For each fraction value, a hundred random error sets were generated and closed orbit, beta functions, and dynamic aperture were calculated. It was immediately found that for the error values equal to those given in Table II, the closed orbit did not exist in $100 \%$ of the cases. Figure 2 shows the percentage of the cases for which a closed orbit exists as a function of the error strength. One can see that even for half the value of errors in Table II, closed orbit exists only for $10 \%$ of the cases. It is worth mentioning here that the absence of closed orbit solution for lattice with the expected errors (values given in Table II) indicates that the evaluation of lattice performance with realistic errors cannot be done

TABLE II. Rms values for various errors used for start-to-end lattice commissioning simulation.

Girder misalignment

Elements within girder

Dipole fractional strength error

Quadrupole fractional strength error

Dipole tilt

Quadrupole tilt

Sextupole tilt
$100 \mu \mathrm{m}$

$30 \mu \mathrm{m}$

$1 \times 10^{-3}$

$1 \times 10^{-3}$

$0.4 \mathrm{mrad}$

$0.4 \mathrm{mrad}$

$0.4 \mathrm{mrad}$ 


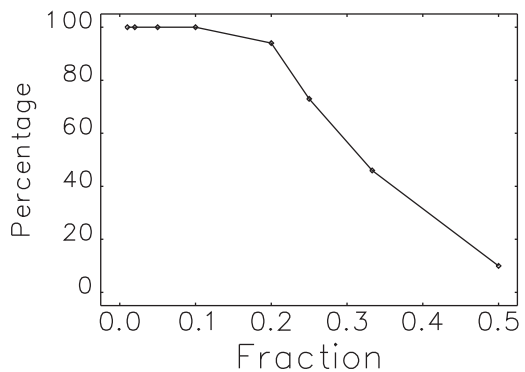

FIG. 2. Percentage of the cases with existing closed orbit solution as a function of the error magnitude expressed as a fraction of the values given in Table II.
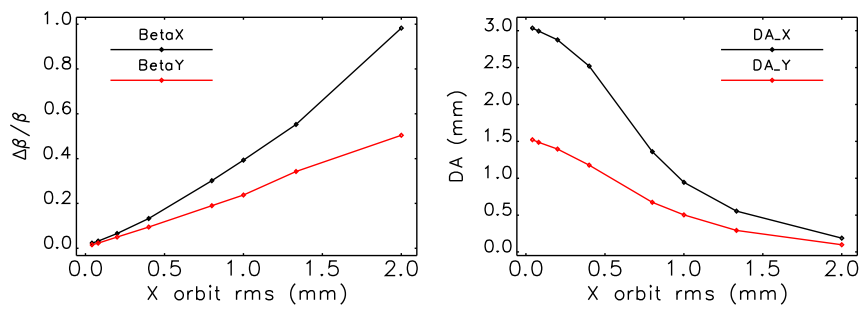

FIG. 3. Rms beta function distortion (left) and median horizontal and vertical dynamic aperture (right) as a function of the horizontal closed orbit errors. The orbit errors correspond to the beginning of the lattice where $\beta_{x}=5 \mathrm{~m}$.

without some sort of commissioning or error ramping procedure.

For the cases where closed orbit exists, beta functions and dynamics aperture were calculated. Figure 3 shows rms relative beta function errors and horizontal and vertical median dynamic aperture as a function of the rms horizontal orbit errors. Figure 4 shows median dynamic aperture for different magnet error values. One can see that when orbit errors exceed $1 \mathrm{~mm} \mathrm{rms}$, the dynamic aperture reduces to less than $1 \mathrm{~mm}$. When the dynamic aperture becomes smaller than the expected orbit errors, correcting trajectory of injected beam might not be enough to capture the beam. In this situation, the distance between the corrected trajectory and closed orbit could exceed the dynamic aperture, and no particles would be captured during injection. This is an important finding that separates newly designed light sources from the previous generation.

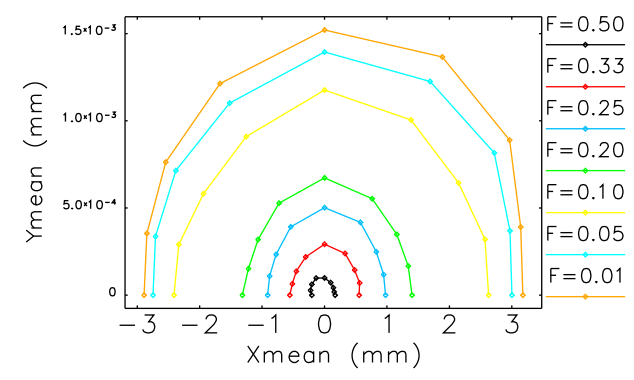

FIG. 4. Median dynamic aperture for different values of magnet errors expressed as a fraction of the values given in Table II.

\section{COMMISSIONING SIMULATION PROCEDURE}

While the effect of individual lattice imperfections on accelerator performance can be estimated or calculated analytically, including all errors together is beyond the realm of analytic estimations. To understand how various errors combine together and impact commissioning, a startto-end simulation of machine commissioning was performed taking into account as many errors as possible. The procedure was programmed using the Tcl/Tk [12] scripting language, with data processing performed using the SDDS toolkit $[13,14]$. All accelerator-related simulations were performed using ELEGANT [15]. The simulated commissioning procedure closely follows the steps that will be performed during actual commissioning, and includes the following major steps:

(i) Generate displacement, tilt, and calibration errors for all elements using Gaussian distributions with $2 \sigma$ cut off.

(ii) Correct the turn-by-turn trajectory until the beam survives for about 20 turns.

(iii) Correct the closed orbit down to an acceptable level; adjust tunes if needed.

(iv) Correct optics and coupling.

(v) Adjust coupling to obtain the required emittance ratio (usually $10 \%$ ).

In this paper we will describe the details of every step and show achieved correction results. At the end, we will give a table that briefly summarizes each correction step.

Before proceeding with the commissioning, the starting quadrupole strengths (i.e., those assumed before the addition of errors) are adjusted to nominally move the betatron tunes away their design values, which are close to integer and coupling resonances. This will reduce the negative effect of these resonances during initial commissioning. The design fractional tunes are 0.12 in both planes; they are adjusted to 0.18 and 0.24 . After commissioning is completed, the tunes are moved back to the design values.

In some stages of the process, tracking of injected beam is performed; this is used to simulate, for example, threading the beam through the first turn. In such cases, the simulation uses a bunch of macroparticles with emittances and energy spread corresponding to the expected parameters of the bunch extracted from the booster at $6 \mathrm{GeV}$, which are given in Table III. To save computing time, the

TABLE III. Phase space parameters of the injected beam.

\begin{tabular}{lcc}
\hline \hline Horizontal emittance & $\epsilon_{x}$ & $60 \mathrm{~nm}$ \\
Vertical emittance & $\epsilon_{y}$ & $6 \mathrm{~nm}$ \\
Horizontal beta function & $\beta_{x}$ & $7 \mathrm{~m}$ \\
Vertical beta function & $\beta_{y}$ & $3 \mathrm{~m}$ \\
Energy spread & $\sigma_{\delta}$ & $1 \times 10^{-3}$ \\
Bunch length & $\sigma_{s}$ & $15 \mathrm{~mm}$ \\
\hline \hline
\end{tabular}


TABLE IV. Physical apertures included in simulations.

Aperture everywhere

$\varnothing 20 \mathrm{~mm}$

ID aperture

ellipse $20 \times 6 \mathrm{~mm}$

Radiation absorbers at every BPM

$\pm 8 \mathrm{~mm}$ in $\mathrm{X}$

bunch consists of only 100 macroparticles. Synchrotron radiation effects are included as a lumped element at the end of the ring. All physical apertures are included, with the values given in Table IV. As was mentioned before, the storage ring will be commissioned with the majority of the small-gap ID vacuum chambers installed.

Table V lists the corrector and beam position monitor (BPM) errors that are included in the simulations in addition to the magnet errors given in Table II. While error generation for element misalignments and magnet strengths and tilts is straightforward, girder misalignment is slightly more involved. The upstream and downstream ends of the girder are randomly misaligned with the displacement given in Table II; the magnets on the girder are assigned displacements following the straight line connecting the ends of the girder (individual element misalignments are added after that). This way of generating girder misalignments closely reproduces the real girder alignment process. This misalignment can also be decomposed into parallel displacement with rms of $x_{\text {girder }} / \sqrt{2}$ and pitch/yaw angle with rms of $2 x_{\text {girder }} /\left(\sqrt{2} L_{\text {girder }}\right)$, where $x_{\text {girder }}$ is the rms girder misalignment given in Table II.

Since the simulation procedure is fully automated, commissioning can be simulated for hundreds of different error sets. The correction results are statistically analyzed for residual orbit and lattice perturbations, correctors strengths, emittances, etc. For each error set, various performance measures (e.g., rms horizontal beta functions error) are computed. These are then histogrammed over all error sets. Below, a detailed description of the commissioning procedure is given.

\section{A. Trajectory correction}

If nonlinear effects coming from sextupoles are neglected, or it is assumed that the sextupoles are turned off at the beginning of commissioning, one can estimate the expected closed orbit error at a particular location assuming

TABLE V. Corrector and beam position monitor errors used in addition to the magnet errors shown in Table II.

\begin{tabular}{lc}
\hline \hline Corrector calibration error & $5 \%$ \\
Initial BPM offset error & $500 \mu \mathrm{m}$ \\
BPM calibration error & $5 \%$ \\
BPM single-shot measurement noise & $30 \mu \mathrm{m}$ \\
BPM orbit low-current noise & $3 \mu \mathrm{m}$ \\
BPM orbit high-current noise & $0.1 \mu \mathrm{m}$ \\
BPM and corrector tilts & $1 \mathrm{mrad}$ \\
\hline \hline
\end{tabular}

independent random misalignments of magnets and girders and using orbit amplification factors calculated using lattice functions or a built-in feature of ELEGANT. Taking the error levels from Table II, rms orbit errors are estimated to be $7 \mathrm{~mm}$ in the horizontal and $4 \mathrm{~mm}$ in the vertical plane at the ID locations. Considering that the full vertical aperture is $6 \mathrm{~mm}$ (see Table IV) and that there are 35 insertion device chambers in the ring, it is entirely possible that the orbit error at one of these chambers would exceed the vacuum chamber gap. Based on this orbit error estimation, it is clear that first-turn trajectory correction will be needed in order to obtain a closed orbit inside the vacuum chamber.

Modern accelerators have the advantage of having accurate turn-by-turn beam-position monitors, which can record the trajectory of the injected beam over several turns. These will be present in the APS upgrade ring and hence are used for first-turn trajectory correction in these simulations.

The first step in first-turn trajectory correction is the correction of the injected beam trajectory at the entrance to the storage ring. It was determined that if the injected beam has a sufficiently good trajectory to get through the Lambertson septum magnet [16], then the beam easily passes through at least one sector in the storage ring. Based on this, the trajectory of the beam in the first sector is used to determine and correct the incoming trajectory. For simulation of the incoming trajectory correction, the expected trajectory errors were calculated based on the geometry of the septum magnet vacuum chamber. Table VI (middle column, labeled "Static errors") gives the errors used in the simulations. Phase error is not included since the storage ring $\mathrm{rf}$ is not turned on at this point.

The calculation of the injected-beam trajectory and its correction is done the following way. The trajectory is measured on all BPMs in the first sector and then tracked back to the beginning of the first sector using ideal transfer matrices, which provides the injected beam position and angle at the beginning of the lattice relative to the ideal orbit. In real life, this data will be used to adjust correctors in the transport line and injection kickers. Since neither transport line nor injection kickers are simulated here, in this study the trajectory position and angle correction are simply subtracted from the known initial errors. The process is repeated for several iterations until it converges. The simulation is usually performed for 200 random error

TABLE VI. Injection trajectory static and shot-to-shot rms variations.

\begin{tabular}{lcc}
\hline \hline & Static errors $(\mathrm{rms})$ & Jitter $(\mathrm{rms})$ \\
\hline Horizontal position & $2 \mathrm{~mm}$ & $100 \mu \mathrm{m}$ \\
Horizontal angle & $0.5 \mathrm{mrad}$ & $10 \mu \mathrm{rad}$ \\
Vertical position & $0.5 \mathrm{~mm}$ & $25 \mu \mathrm{m}$ \\
Vertical angle & $0.3 \mathrm{mrad}$ & $15 \mu \mathrm{rad}$ \\
Energy & $0.5 \%$ & $10^{-4}$ \\
\hline \hline
\end{tabular}



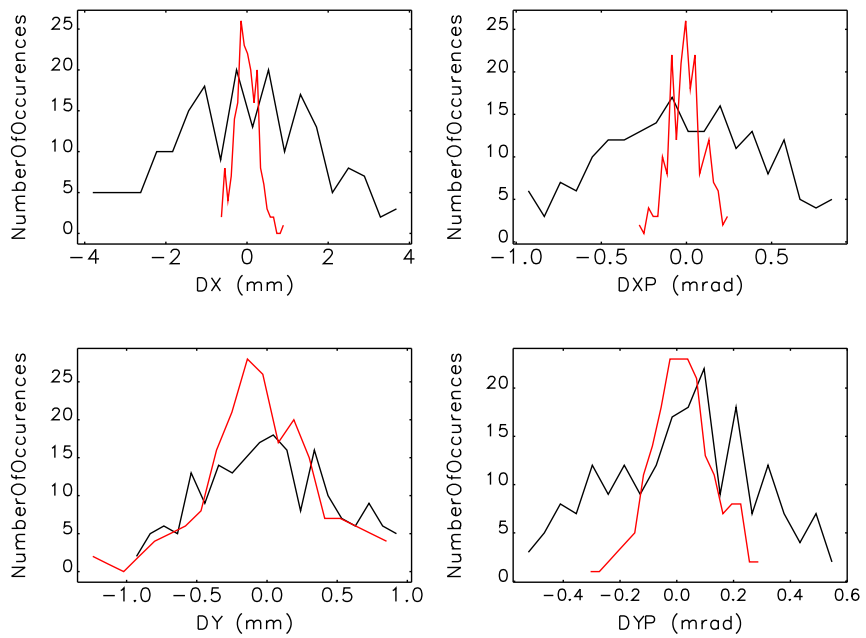

FIG. 5. Histograms of the injection trajectory error before (black) and after (red) correction at the beginning of the lattice. DX/DY stand for the horizontal/vertical positional errors, while DXP/DYP stand for the horizontal/vertical angular errors.

sets (all statistical analysis presented in this paper is based on simulation of 200 random error seeds if not mentioned otherwise). BPM offset and calibration errors as well as single-shot measurement noise from Table $\mathrm{V}$ are taken into account when calculating the injected-beam trajectory in the first sector. Figure 5 shows the results of the injected trajectory correction, which results from several iterations of the correction. Black lines show the histograms before correction, while red lines give the results after the correction. DX/DY stand for the horizontal/vertical positional errors, while DXP/DYP stand for the horizontal/ vertical angular errors. It can be seen that the trajectory correction converges to residual errors of about $0.5 \mathrm{~mm}$ and $0.1 \mathrm{mrad} \mathrm{rms}$. At this point, the energy error cannot yet be determined.

After the trajectory of the injected beam is corrected, one can take a first look at how far along the ring the injected beam can go without any further corrections. Figure 6 shows the cumulative distribution function $(\mathrm{CDF})$ of the

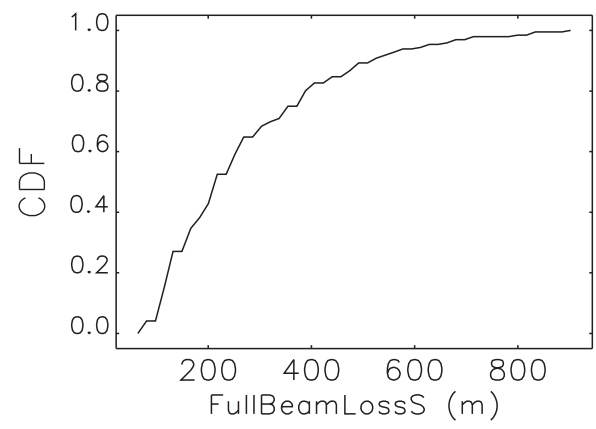

FIG. 6. Cumulative distribution function of the beam loss position after correction of the injection trajectory only (no trajectory correction in the ring). position of full beam loss (defined as the point where only $10 \%$ of the initial beam is left). One can see that for half the cases the beam is lost completely before traveling 300 meters along the ring circumference, while in all cases the beam is lost before reaching 900 meters. This means that the beam does not reach the end of the first turn.

\section{Injection jitter}

In addition to static injected beam trajectory errors described earlier, there is also a shot-to-shot variation, or jitter, which cannot be corrected. Injection into the storage ring [17] is performed in vertical plane using a DC Lambertson septum magnet, and therefore the horizontal jitter is dominated by the two booster extraction pulsed magnets; measurement results for this jitter were reported in $[18,19]$. Each magnet has a uniform noise distribution, and together they combine to provide quasi-Gaussian noise with $5 \mu \mathrm{radrms}$. Design of the booster-to-storage ring (BTS) transport line is still in progress, but one can use the beta functions at the extraction and injection points and assume conservatively the worst possible phase advance factor to calculate how the initial angle jitter transforms into position and angle jitter at the injection point. Booster extraction energy variation was also measured $[18,19]$.

The measured vertical trajectory variation in the transport line is very small, so jitter in the vertical plane will be dominated by the storage ring vertical injection kickers. The assumed vertical angle jitter was calculated based on bench measurements of the test power supply. Table VI (right column) gives the rms jitter numbers with $50 \%-100 \%$ safety factor included.

\section{First-turn correction}

According to the simulations shown in Fig. 6, the beam is not expected to achieve first-turn transmission without correction. Therefore, the first step of the trajectory correction is to advance the beam to the end of the first turn. Several methods were tested for the first-turn trajectory correction: "one-to-next" correction when steering is performed by pairing one corrector with the next downstream BPM; "one-to-best" correction attempts to find a BPM with a large response to each corrector; "threading" does corrector sweeps to work the beam through a beamline; and "global" correction that simply uses the global trajectory response matrix. All these methods suffered from having relatively weak orbit correctors. It was mentioned before that due to the compactness of the lattice, the corrector strengths are rather limited. Table VII shows the design corrector strength limits. If one considers that $1 \mathrm{~mm}$ trajectory error in a single Q8 quadrupole (the strongest quadrupole) produces a $2.3 \mathrm{mrad}$ kick, it is clear that correction methods that attempt to utilize individual correctors have little chance of succeeding in achieving the first-turn transmission. 
TABLE VII. Design corrector strength limits.

Standalone fast correctors

S1 sextupole orbit correcting coils

Q7 quadrupole orbit correcting coils

Q8 quadrupole orbit correcting coils

Figure 7 shows beta functions and location of BPMs and correctors within one sector. All correctors are combined horizontal and vertical correctors. Overall, there are 10 correctors and 14 BPMs per sector arranged approximately symmetrically around the middle of the sector. BPMs are named from P0 to P6 in each half of the sector. BPMs P2 and $\mathrm{P} 4$ are not single-turn capable and therefore not used for trajectory and initial orbit correction.

Since real commissioning will be performed with the vast majority of ID chambers already installed, and since the ID chambers represent the limiting apertures in the ring, the beam will most likely always be lost on an ID chamber. Hence, the procedure for threading the beam through the first turn attempts to correct the beam trajectory at every ID chamber. A virtual BPM in the middle of the ID vacuum chamber is created that utilizes three real BPMs on each side of the ID chamber to calculate position and angle of the trajectory in the middle of the ID chamber. This calculation assumes ideal transfer matrices between BPMs, and only BPMs with sufficient beam present (i.e., sufficient sum signal) are used in the calculation. To overcome strength limits of individual correctors, 10 correctors in the sector immediately upstream of the ID chamber are used simultaneously to reduce both the position and angle of the beam at the location of the virtual BPM. The corrector strengths are calculated using singular value decomposition of the ideal trajectory response matrix.

For 200 error sets, this procedure fails to advance the beam through at least one ID chamber for about $15 \%$ of the error sets. The failure usually happens when the algorithm gets large errors in the determination of the position and angle, which in turn happens when only a few BPMs out of six see the beam. For these cases, a simplex optimization is run that uses the same correctors to vary horizontal and

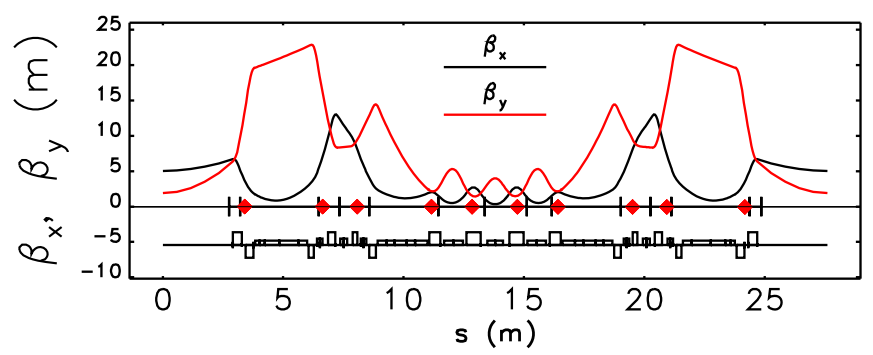

FIG. 7. Beta functions with locations of BPMs and correctors. Correctors are shown as red diamonds on the horizontal axis, and BPMs are vertical lines on the same axis. Lattice elements are also shown below the horizontal axis.

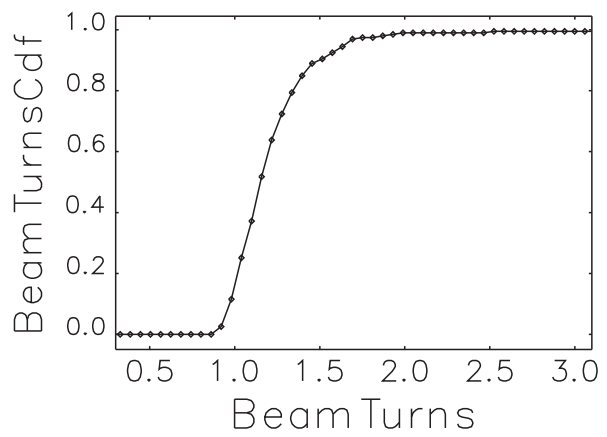

FIG. 8. Cumulative distribution function of the beam-turns integral after first-turn beam threading.

vertical position and angle in an attempt to maximize the beam transmission, which is measured as an integral $\int N_{\text {particles }}(s) d s$ normalized by the initial number of particles and the circumference of the ring. This value represents "beam-turns" integral and is used throughout the commissioning procedure and this article. Figure 8 shows CDF of the beam transmission after first-turn threading. The beam at this point successfully reaches the end of the first turn for all error sets but does not go beyond the second turn.

\section{Static injected beam energy error correction}

As was mentioned before, the error of the injected beam energy has not been determined yet. It was found that the average trajectory on all BPMs can serve as a good indication of the injected beam energy error. The following steps are performed to correct the energy error: when the beam threading step reaches half way around the ring, the energy error is calculated as the ratio of the average horizontal trajectory to average ideal horizontal dispersion function on all BPMs $-\Delta E / E=\langle x\rangle /\left\langle\eta_{x}\right\rangle$. The energy of the injected beam is offset by the negative of this value, then beam threading is restarted from the beginning. Figure 9 shows the histogram of the energy error before and after correction. $\Delta E / E$ accuracy of about $10^{-3} \mathrm{rms}$ can be achieved.

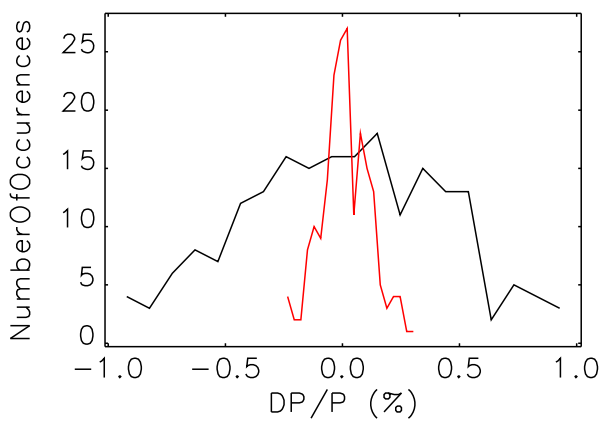

FIG. 9. Histogram of the beam energy of the injected beam before (black) and after (red) correction. 


\section{BPM verification}

Despite the extensive effort that is being planned to spend on the in-tunnel BPM verification, there still exists a chance, though very small, of connecting a BPM the wrong way. If some BPM buttons are misconnected or a button or its connecting cable is damaged, it will manifest itself in a large BPM gain error, large horizontal-vertical coupling, or a large offset error. These BPM errors can further complicate the trajectory correction. To avoid this complication, a beam-based BPM check out is run while the first-turn trajectory threading is performed.

The BPM verification is done the following way. After the beam has been threaded through an ID vacuum chamber, it passes through entire next sector. At this point, a trajectory response matrix is measured using four correctors in each plane (two correctors immediately upstream of this sector and two correctors at the beginning of the sector) and all BPMs in the sector of interest. Then the trajectory response matrix fit is performed to determine the BPM gain and tilt errors. Four correctors in each plane provide enough data to determine BPM gain errors with an rms accuracy of about $15 \%$ and tilt angle errors with an rms of $10 \mathrm{deg}$. The main limitation on the accuracy of the BPM error determination at this point is the injection jitter and BPM noise. Such accuracy is suitable for diagnosing BPM cable connection errors.

The BPM verification process slows the first-turn trajectory correction significantly by adding almost 300 injections required to measure the trajectory response matrices. The process will be further optimized with the goal of reducing the number of required injections. The same approach could also be used for searching grossly miswired magnets, but it has not been tested yet.

\section{Improvement of the multiturn beam transmission}

After first-turn transmission is achieved, one can imagine that if the beam coordinates at the end of the first turn are made exactly the same as at the entrance into the ring, this would constitute a closed orbit. It was found in these simulations that this approach works reasonably well. We also tested a simplex optimization of the beam trajectory at the end of the first turn to maximize the beam-turns integral. This simplex optimization is exactly the same as was described in the beam threading section. Figure 10 shows the improvement of the beam-turns integral after using simplex and equalizing the coordinates at the end of sector 40 to the coordinates of the injected beam. One can see that the 50th percentile beam-turns integral is increased from 1.0 to 2.2 beam-turns as the result of this step. The transmission improvement is clear, but not very impressive. It is also interesting to note, that making beam trajectory at the end of sector 40 equal to the trajectory of the injected beam gives approximately the same transmission improvement as the simplex optimization, though simplex takes much longer to optimize the trajectory.

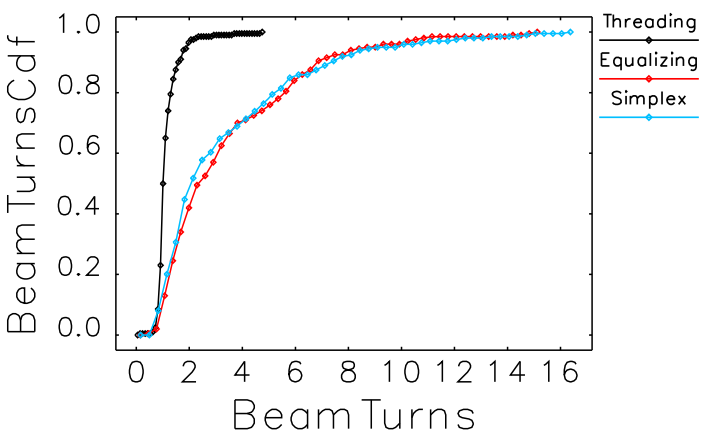

FIG. 10. Cumulative distribution function of the beam-turns integral after first-turn threading (black), after equalizing beam trajectory at the end of sector 40 to the injected trajectory (red), and after simplex optimization of the beam trajectory at the end of sector 40 .

\section{6. $r f$ setup}

As shown in Fig. 10, after equalizing beam trajectory at the end of sector 40 to the coordinates of the injected beam, some fraction of the beam is likely to survive at least 5 turns. To proceed further, the accelerating rf voltage needs to be turned on, and its phase and frequency need to be set correctly. As was mentioned earlier, the average orbit error on all BPMs serves as a good indicator of the relative energy error. This way of measuring beam energy error is used to determine rf phase and frequency errors. Presently, the rf system is considered to be a single cavity, meaning that possible cavity-to-cavity phase errors are not taken into account.

The beam energy change on turn $n$ can be expressed as difference between the energy gained from rf and that lost to synchrotron radiation:

$$
\begin{aligned}
\delta E_{n} & =e V \sin \left(\left(\omega_{0}+\Delta \omega\right) T_{0} n+\phi_{0}+\Delta \phi\right)-U_{0} \\
& =e V \sin \left(\Delta \omega T_{0} n+\phi_{0}+\Delta \phi\right)-U_{0},
\end{aligned}
$$

where $T_{0}$ is the turn length, $\omega_{0}=2 \pi / T_{0}, \phi_{0}$ is synchronous phase, $\Delta \omega$ and $\Delta \phi$ are frequency and phase errors, and $V$ is the rf voltage. In this expression, the energy-dependent path-lengthening was neglected due to the small momentum compaction factor of the lattice $\left(\alpha_{c} \approx 4 \times 10^{-5}\right)$ and short tracking time (20-50 turns compared to a synchrotron period of about 300 turns). Integration of (1) gives the full beam energy as the function of turn number $n$ :

$$
\begin{aligned}
E_{n}= & \tilde{E}-\frac{\mathrm{eV}}{\Delta \omega T_{0}}\left(\cos \left(\Delta \omega T_{0} n+\phi_{0}+\Delta \phi\right)\right. \\
& \left.--\cos \left(\phi_{0}+\Delta \phi\right)\right)-n U_{0}
\end{aligned}
$$

where $\tilde{E}$ is the initial energy of the beam and the term $-\cos \left(\phi_{0}+\Delta \phi\right)$ was introduced to make $E_{0}=\tilde{E}$. It is assumed that the initial beam energy is equal to the 
equilibrium beam energy because the beam energy was set up in the prior steps when the rf was still off.

The average orbit error on BPMs gives the relative energy deviation from the equilibrium energy $\Delta E / E$. Expression (2) can be rewritten in relative terms:

$$
\begin{aligned}
\frac{\Delta E_{n}}{E}= & -\frac{\mathrm{eV}}{E \cdot \Delta \omega T_{0}}\left(\cos \left(\Delta \omega T_{0} n+\phi_{0}+\Delta \phi\right)\right. \\
& \left.--\cos \left(\phi_{0}+\Delta \phi\right)\right)-n \frac{U_{0}}{E} .
\end{aligned}
$$

This expression can be used to fit the measured $\Delta E_{n} / E$ using variables $\Delta \omega$ and $\Delta \phi$. The effect of a voltage error can be subsumed into the $\Delta \phi$ variable for now.

As an example, Fig. 11 shows the beam energy as a function of turn number in a case with no beam losses. For this example, the following errors were introduced: $\Delta \phi=-35^{\circ}$ and $\Delta \omega=-8 \mathrm{kHz}$. The fit is done using sddsgenericfit (part of the SDDS toolkit). It was found that sometimes sddsgenericfit is not able to find the best fit due to large initial errors of the fit parameters. This was resolved by splitting the total variation range for $\Delta \omega$ into several smaller regions, running the fit in each region separately, and then choosing the best fit.

When the beam only survives for a few turns, there is no way to distinguish between the phase and frequency errors. Therefore, the initial rf setup is only done for the phase. First, the correct phase slope is set by calculating the energy slope with respect to turn number for different phase settings. If the phase slope is wrong, the phase is changed by $45^{\circ}$ until the correct energy slope is achieved. After that, a coarse phase adjustment is performed using the simplified expression (1) when $\Delta \omega$ is set to zero. Later in the correction procedure when the beam transmission exceeds 10 turns, the beam energy fit is switched from the simplified version of Eq. (1) with one variable (phase) to the full expression in Eq. (3) with two variables-phase and frequency.

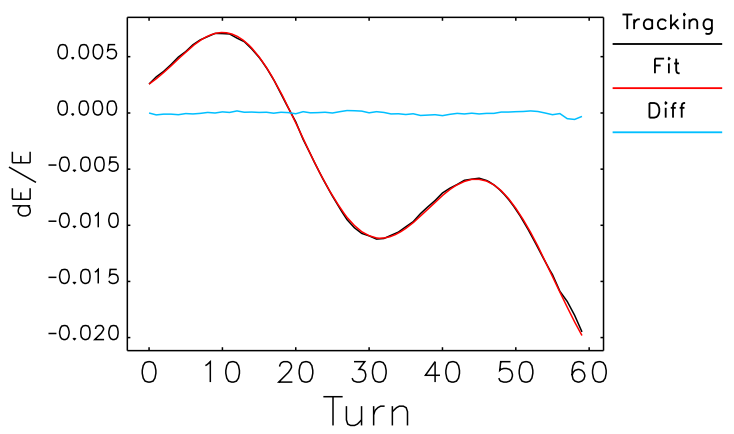

FIG. 11. Beam energy oscillation and its fit for $\Delta \phi=-35^{\circ}$ and $\Delta \omega=-8 \mathrm{kHz}$. Black line-tracking, red line-fit, blue linedifference between tracking and fit.

\section{Global trajectory correction}

Early in the commissioning study, it was realized that the required orbit corrector strengths could easily exceed the corrector limits. Therefore, one of the goals of the trajectory correction procedure was to minimize the required corrector strengths. Once a full turn is achieved, it becomes possible to use a global trajectory correction algorithm, which can "iron out" locally strong steering.

Global trajectory correction is performed using the ideal trajectory response matrix. The correction consists of three nested loops. The outer loop is a loop over different corrector configurations starting from small number of correctors per sector and increasing. For every corrector configuration, the correction is run with the trajectory response matrix inverted using a different number of singular values (SV), starting with small SV number and increasing it - this is the second loop. For every fixed SV number, the correction is run repeatedly until convergence is achieved-this is the third loop.

At every step, the best trajectory is recorded. Also, if any corrector reaches a specified fraction of the limit at any step, it is excluded from further correction. After every iteration of the inner loop, the adjustment of the rf phase and frequency is performed as described in Sec. III A 6. The correction is completed when the target beam-turns integral is achieved or when all the loops are completed (the target is usually set to between 15 and 20 beam-turns).

The transmission achieved after running this procedure with the beam-turns integral target set to 20 is shown in Fig. 12. One can see that for about half the cases the beam transmission is limited to less than 10 turns despite having the trajectory corrected as well as possible given the uncertainty in the BPM offsets.

It is interesting to note that for most of the cases shown in Fig. 12 the simulation program does report the existence of closed orbit. However, tracking shows that the injected particles are not captured on this orbit. It is exactly the situation that was predicted in Sec. II: when the distance between the corrected trajectory and closed orbit exceeds

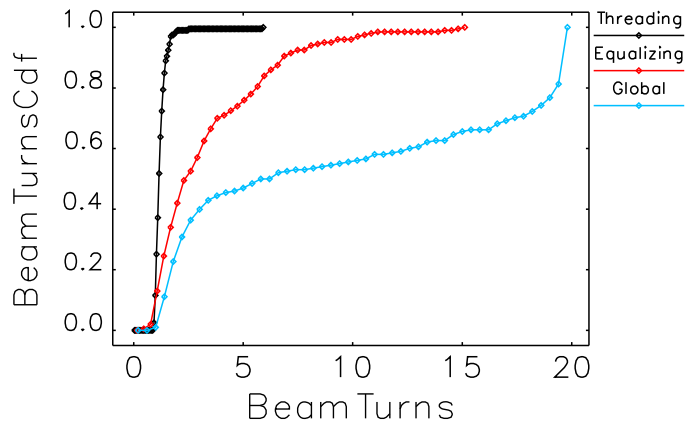

FIG. 12. Cumulative distribution function of the beam transmission in beam-turns after first-turn threading (black), equalizing trajectory at the end of the first turn (red), and global trajectory correction (blue). 


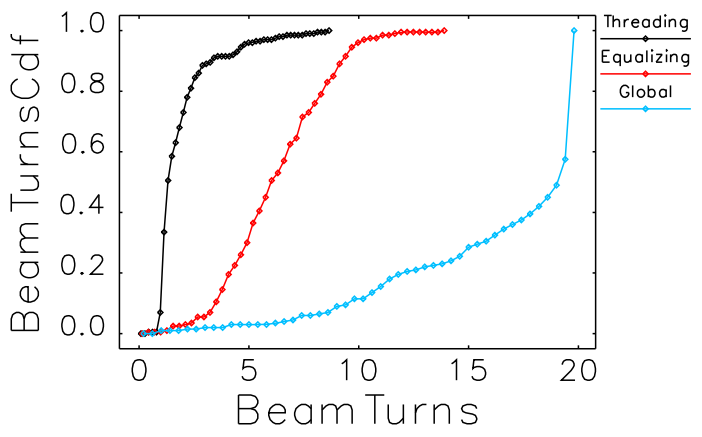

FIG. 13. Cumulative distribution function of the beam transmission with sextupoles turned off. The same cases as in Fig. 12 are presented.

the dynamic aperture, injected particles do not get captured even after the trajectory correction is complete. The solution for this problem is simple - turning off sextupoles should increase the dynamic aperture. Figure 13 gives the beam transmission results when the sextupoles are turned off before commissioning. One can see that for about $90 \%$ of cases the beam survives longer than 10 turns. The comparison of Figs. 12 and 13 makes it clear that the sextupoles should be turned off before commissioning. In retrospect, one might say that the need to start with sextupoles off right away was obvious based on what was discussed in Sec. II. However, most of the synchrotron light sources commissioned to date did not need this. In addition, if one started with the sextupoles turned off, turning them on in a strongly focusing lattice is also not an easy task. This motivated the initial choice to start with sextupoles turned on.

Figures 14 and 15 show the final results of trajectory correction. Figure 14 shows the CDF of the number of injection shots required to pass each step of the trajectory correction procedure: first-turn threading, equalizing trajectory coordinates at the end of the first turn to the injected beam coordinates, rf setup, and global trajectory correction. This plot basically tells the time required to achieve the multiturn transmission. For example, it takes

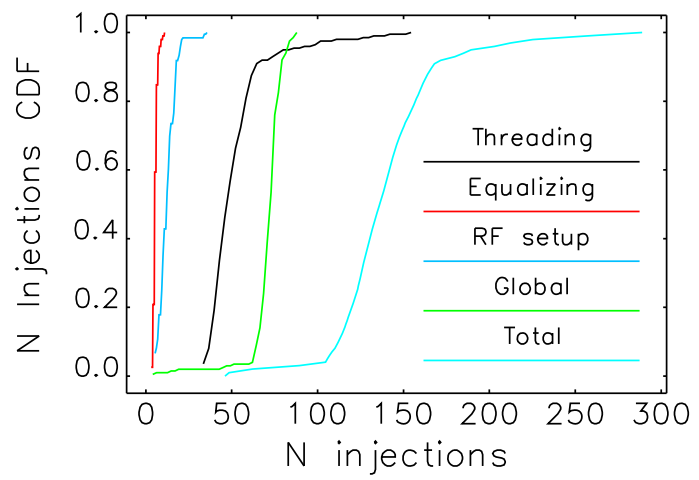

FIG. 14. CDF of the number of injections required to pass each trajectory correction step.

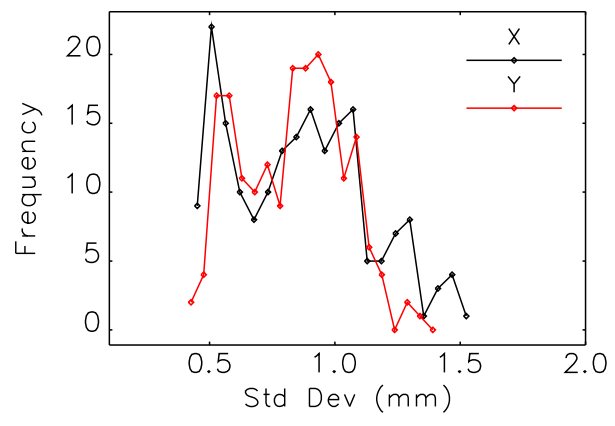

FIG. 15. Histogram of standard deviation of the trajectory relative to the design orbit after completion of the trajectory correction step.

about 150 injection cycles to achieve multiturn transmission for the median case; if one assumes that an injection shot and subsequent processing takes 5 seconds, then multiturn transmission should be achieved in about 13 minutes. This time, however, does not include the BPM verification process that could actually triple the number of required injections, as was discussed Sec. III A 4.

Figure 15 shows the histogram of the rms trajectory error after completion of the trajectory correction. The histogram is calculated the following way: for each error set, the rms of the trajectory error over all BPMs relative to the design orbit is calculated, then the distribution of those rms values over all error sets is presented as a histogram. The average rms trajectory error over all error sets is $0.7 \mathrm{~mm}$ for horizontal and $0.8 \mathrm{~mm}$ for vertical trajectory. The accuracy of trajectory correction is mostly limited by the BPM offset errors $(0.5 \mathrm{~mm} \mathrm{rms})$, corrector strength does not limit the correction.

\section{B. Orbit correction}

After the trajectory correction procedure is completed, the commissioning program switches to orbit correction. The beam is still injected and tracked in exactly the same way as was done for trajectory correction, but now the multiturn trajectory is used to calculate the orbit by averaging turn-by-turn readings on every BPM over several turns (only those turns with enough surviving particles are used for averaging). Orbit correction consists of the same three nested loops as was described in the trajectory correction procedure, only this time the nominal orbit response matrix is used instead of the trajectory response matrix. Also, more corrector configurations are used in the outer loop.

After every iteration of the inner loop, the betatron tunes are corrected using the quadrupole doublets bracketing the ID straight sections. Tune correction at this early stage primarily takes care of the initial tune error of the lattice, and it becomes really necessary during the sextupole ramp, which will be described later. Since the beam only survives several turns at this point, the tunes are measured using the 
trajectory response. Instead of fitting the trajectory response, in which case one would have to deal with beta function errors, the trajectory is treated as betatron motion and analyzed similarly to the way fast-kick-induced betatron motion is analyzed to get the tunes. Usually, the turnby-turn motion on a BPM is analyzed using fast Fourier transform (FFT) or numerical analysis of fundamental frequencies (NAFF) [20] to obtain the noninteger part of the tune for one turn. While in our case the number of turns that the beam survives is very small, one turn consists of 40 sectors that are nominally identical, so one can analyze the motion on a sector-by-sector basis using BPMs that are located at the same position in every sector. The tune obtained this way is the noninteger part of the betatron tune per sector, and the full-turn tune can be found by multiplying this per-sector tune by the number of sectors and adding a proper integer. Of course, the phase advance between sectors is not constant due to different errors in each sector, which leads to a broader spectral line and reduced accuracy. Multiplying by the number of sectors also increases the tune determination error. On the other hand, the tunes found this way provide information on the integer part of the tune, and they do not suffer from ambiguity as to whether the full-turn tune is above or below the integer. This is based on the assumption that the integer part of the tune per sector is always the same as the design value, because the errors for the per sector tune are always small (indeed, even if one imagines a total tune error of 4 units, it still only means an error of 0.1 for the per sector tune). This approach was tested in simulations and it was found that an rms accuracy of about 0.05 can be achieved for the full-turn tunes. Before settling on this method, the simple standard approach of taking NAFF of turn-by-turn data on every BPM and then averaging over BPMs to improve accuracy was also tested. It was found that this method gives comparable rms accuracy of about 0.06 , but it also requires more turns before it can be successfully applied. One reason why this method does not provide higher accuracy is motion decoherence due to uncompensated chromaticity- the rms tune spread inside the beam after just one turn is more than 0.1 .

After the first two iterations of the outer loop or when the orbit errors are reduced below a predefined value, the sextupole strengths are gradually ramped up to full strength. The ramp is done in 10 steps, where the ramp steps are performed every few iterations of the inner loop but only if the beam transmission does not drop below a certain number of beam-turns. If the transmission is reduced as a result of the sextupole ramp step, the next sextupole step is not performed until the transmission been sufficiently restored. If at any point the transmission drops below some predetermined number of turns, the procedure returns to trajectory correction and attempts to recover transmission in trajectory mode. The rf phase and frequency adjustment is still being run after every iteration.

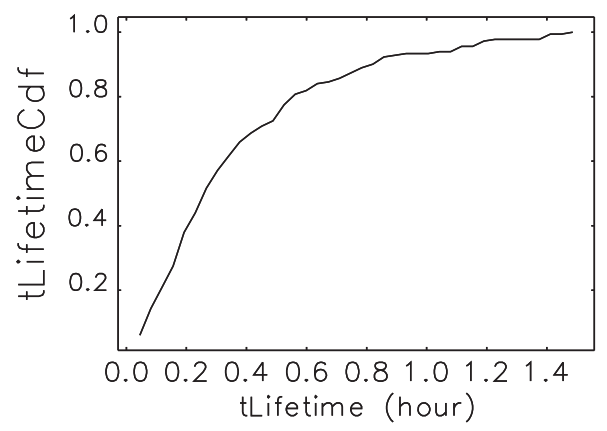

FIG. 16. Lifetime CDF after completion of the sextupole ramp. Tenth percentile lifetime is 0.08 hours, while median lifetime is 0.26 hours.

After the sextupole ramp is completed, the lifetime is estimated using the local momentum aperture and the actual emittance ratio at that moment. The lifetime calculation is rather primitive, as it uses only a few points along the circumference for the local momentum aperture calculation, in order to save computation time. Figure 16 shows the resulting lifetime for a 1-mA bunch. It is worth noting that having uncorrected coupling errors and large vertical beam size at this point helps in achieving reasonable lifetime. The estimated lifetime at this point is large enough so that one could perform beam-based measurement of the BPM offsets. The BPM offset measurement is simple enough, so it is not simulated here. The offset errors are simply reduced from $500 \mu \mathrm{m}$ to $30 \mu \mathrm{m} \mathrm{rms}$ relative to nearby quadrupoles. Also, since the transmission is now achieved for many turns, one can safely switch from closed orbit calculation based on multiparticle tracking to the closed orbit calculation inside ELEGANT, which is much faster.

The orbit is calculated by ELEGANT with the assumption of constant path length. Under this condition, the beam energy after orbit correction may have some error that is defined by the integral of all errors of horizontal dipole fields (coming from misaligned magnets and orbit correctors). This error will partially manifest itself in a nonzero average horizontal orbit error. In actual commissioning this will be corrected by adjusting the rf frequency. In the simulations, the rf frequency is tied to the ideal circumference of the ring, so the simulation program instead adjusts the orbit path length using a misalignment (MALIGN) element in ELEGANT to reduce the average horizontal orbit error to zero. Figure 17 shows the beam energy error after commissioning with and without correction of the horizontal orbit average error. One can see that correcting the average orbit error reduces the beam energy error only by about $30 \%$, and the expected rms energy error due to dipole errors and orbit correction is about $5 \times 10^{-4}$.

At the end of every iteration of the outer loop (the loop over the corrector configurations), a coarse optics 


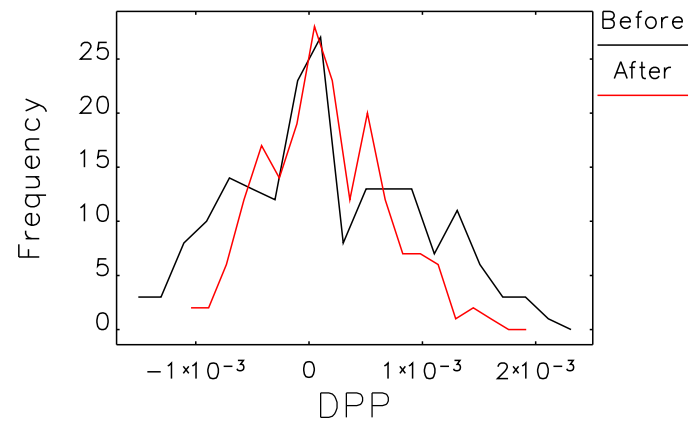

FIG. 17. Beam energy errors before and after correction of the horizontal orbit average error. The corresponding rms variations are: $\sigma_{\text {before }}=8.2 \times 10^{-4}$ and $\sigma_{\text {after }}=5.4 \times 10^{-4}$.

correction is performed that is based on analysis of turn-byturn motion following a fast kick. This measurement is fast and can be performed even when the beam only survives a few dozens of turns. Kicks of $0.5 \mathrm{mrad}$ in horizontal plane and $0.1 \mathrm{mrad}$ in vertical plane are used. The measurement uses the simple fact that the maximum oscillation amplitude on a BPM is proportional to the square root of the beta function at that BPM. This approach is complicated by BPM calibration errors, and normally the turn-by-turn based optics correction also requires the measurement of the oscillation phases on every BPM to obtain BPMcalibration-independent measurements [21]. In our case, the BPM calibration errors are expected to be less than $10 \%$ while the optics errors could be many times this. Therefore, the existence of BPM calibration errors is simply ignored during beta function calculation. Only a few tens of turns are required to resolve such coarse modulation of the betatron oscillation amplitude, and therefore the decoherence of the oscillations has little effect on the measurement. An unknown factor in this measurement is the average beta function on all BPMs. Since the betatron tunes of the lattice are close to the ideal tunes (owing to previous tune correction), it is assumed that the average inverse beta functions are equal to those of the ideal lattice. For simplicity, only three BPMs per sector are used for this measurement and one quadrupole per sector is used for correction; this turns out to be enough to reduce beta function beating in most of the cases.

Figure 18 shows the estimated Touschek lifetime after completion of the orbit correction step: with $90 \%$ certainty the lifetime is above 0.28 hours, while the median lifetime is 0.5 hours. Final rms orbit errors are shown in Fig. 19, top; the correction achieves about $80 \mu \mathrm{m} \mathrm{rms}$ orbit error, which most likely is dominated by the $100-\mu \mathrm{m}$ girder misalignment. Beta function errors are shown in Fig. 19, middle plot. The beta beating is already relatively small because the kick-based beta function correction was run several times during orbit correction. The bottom plot in Fig. 19 shows a histogram of the resulting horizontal and vertical emittances.

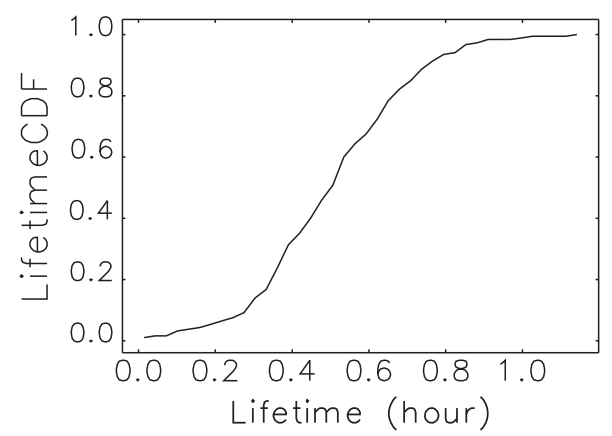

FIG. 18. CDF of lifetime after orbit correction for 1-mA bunch.

\section{Lattice correction}

After the orbit correction is complete, the lifetime is long enough to start detailed lattice correction. A standard correction procedure based on the response matrix fit [22] that was developed for the present APS is used [23]. First, a
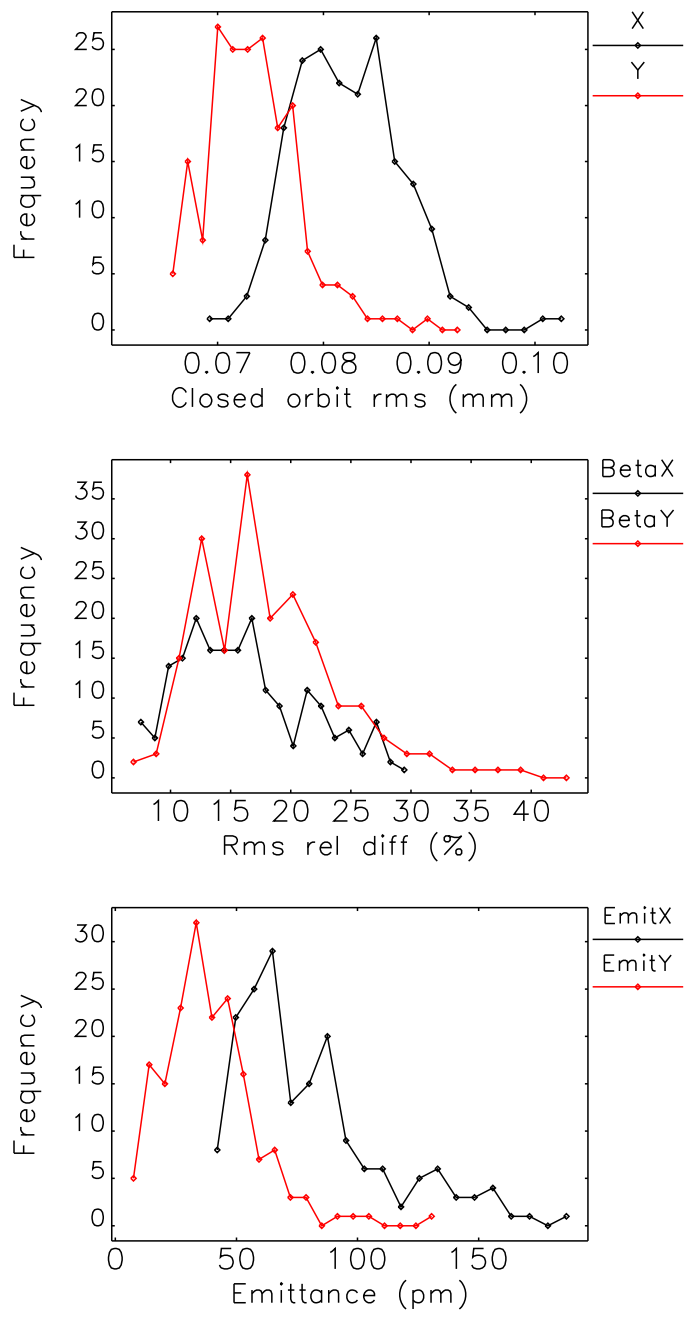

FIG. 19. Results following orbit correction and coarse kickbased lattice correction. Top: Distribution of standard deviation of orbit after correction calculated over 200 random error seeds. Middle: Histograms of rms relative beta function errors after orbit correction. Bottom: Histogram of emittances. 
response matrix measurement is simulated, with BPM noise, BPM and corrector calibration errors, and BPM and corrector tilt errors included. For measurement and calculation speed, only ten correctors per plane are used. Based on the present experience, the measurement time should not exceed five minutes. After the response matrix is generated, the response matrix fit is run to derive focusing and coupling errors.

Beta function and horizontal dispersion correction is calculated using the ideal beta function response matrix (rather than directly using inverse quadrupole errors from the response matrix fit), as this allows for simple correction strength and fraction control using different numbers of singular values in the matrix inversion. Coupling is corrected by minimizing the cross-plane orbit response matrix together with vertical dispersion. All quadrupoles are used for beta function correction, and four skew quadrupoles per sector are used for coupling minimization. Lattice and coupling correction is performed in several iterations while increasing the number of singular values. After every iteration, orbit correction is also performed. Figure 20 shows the results of the beta function correction. The difference in the final accuracy of the correction between the horizontal and vertical planes is about a factor of two. The difference is most likely determined by the difference in the number of BPMs per unit phase advance, but it was not studied in detail and will require further investigation. Figure 21 shows the horizontal and vertical emittances after the correction. One can see that an emittance ratio of $1 \%-2 \%$ can be achieved.

After lattice and coupling correction is complete, the coupling is adjusted to achieve a target emittance ratio of 0.1 by exciting the nearest difference resonance using skew quadrupoles. Then, the tunes are returned to original values (they were detuned further from the integer resonance to make commissioning easier), and chromaticity is adjusted to the design values. Since the original tune point is very close to a difference resonance, the emittance ratio becomes close to 1 , as intended for most of the operating modes. If smaller emittance ratio is required, it can be achieved by

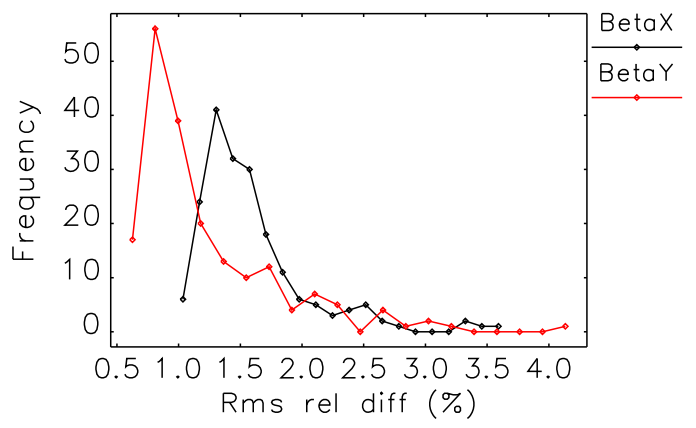

FIG. 20. Distribution of rms beta function errors after lattice correction.
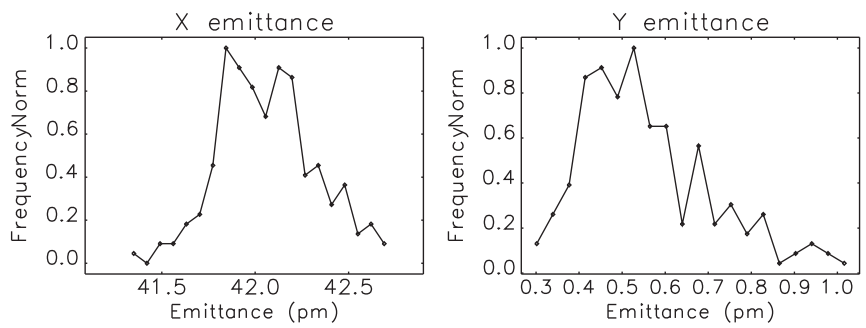

FIG. 21. Histograms of horizontal (left) and vertical (right) emittance after lattice correction.

simply moving the tunes farther away from the coupling resonance.

\section{CONCLUSIONS}

An automated commissioning procedure was written that allows for realistic simulation of storage ring commissioning with many sets of random errors. Table VIII briefly summarizes all steps and gives goals, algorithms, performance, etc for each step. It was found that without trajectory correction, the beam is not expected to complete the first turn due to strong focusing and small vacuum chamber. It was also found that correcting beam trajectory would likely not be enough to capture the beam, because the dynamic aperture is anticipated to be smaller than the typical expected distance between the corrected trajectory and closed orbit. To overcome this difficulty, it is suggested to start commissioning with sextupoles turned off in order to increase the dynamic aperture and make the multiturn beam capture possible.

Initially, the main goal of this work was to demonstrate the possibility of fast commissioning of the APS upgrade lattice, and it was shown that the automated commissioning was successful in $95 \%$ of cases, ensuring fast commissioning of the real storage ring. However, this procedure has found much wider use for the APS upgrade project. The sets of commissioned lattices are now used for evaluation of various effects, such as nonlinear dynamics, beam loss distributions, and collective instabilities in the presence of realistic errors.

The nonlinear dynamics evaluation of APS-U candidate lattices is now done through "ensemble evaluation," when a lattice goes through commissioning simulation which generates 200 commissioned lattices, which are then used to calculate cumulative distribution functions of expected lifetime and dynamic aperture [3], as well as injection efficiency [24]. This ensemble evaluation ensures that only lattices robust against errors are considered. The analysis of the ensemble evaluation results have shown that there exists correlation between the lattice correction accuracy and the lifetime [25]. The commissioning simulation procedure will be used to find ways to improve the accuracy of the lattice correction to reduce variation in the resulting lifetime. The commissioned lattices were also 


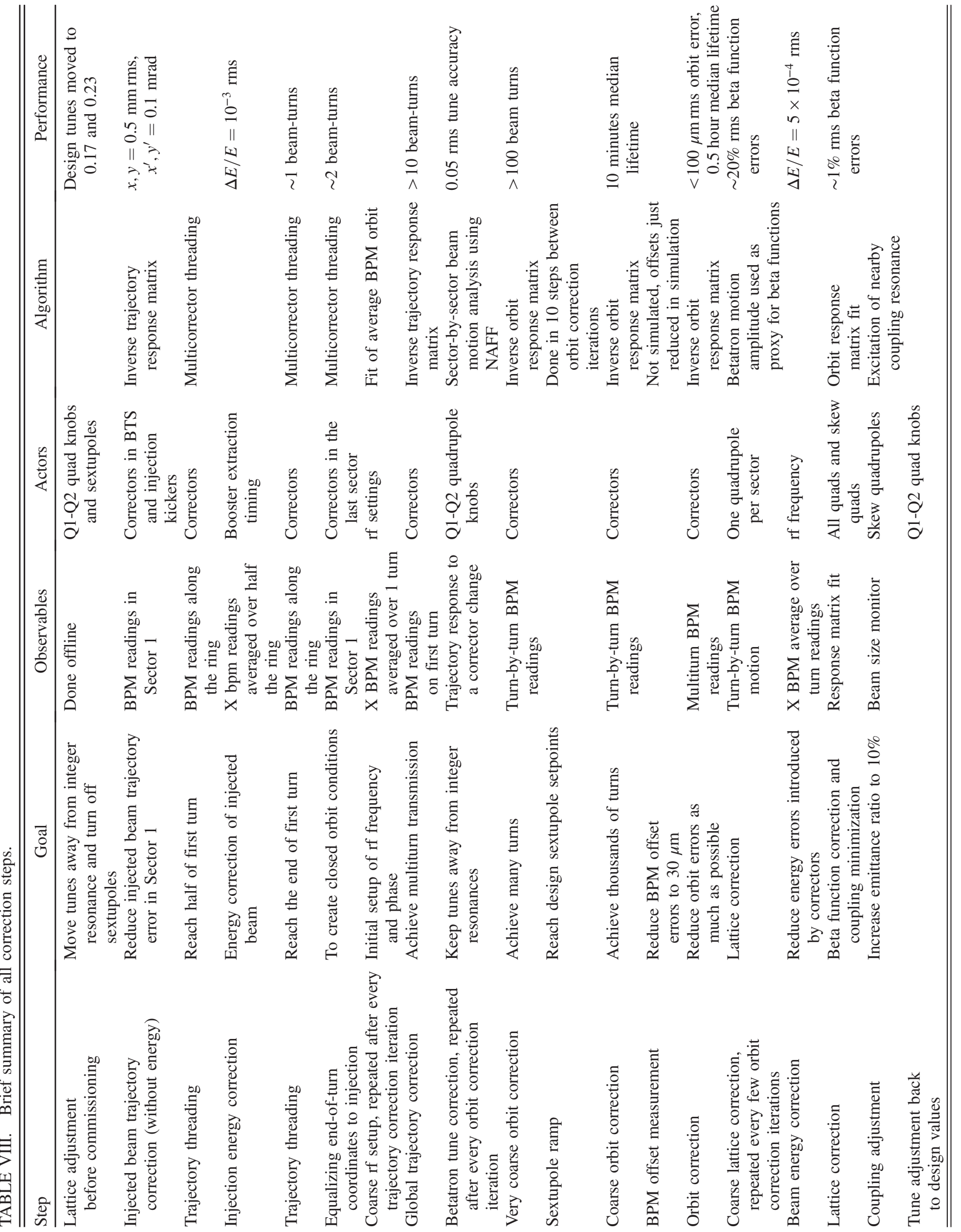


used in accumulation limit studies where it was found that the variation of the equilibrium beam energy coming from dipole errors affects the injection efficiency of high-charge bunches [26].

In addition to helping define error tolerances, the commissioning simulations were used to determine the maximum strengths of dipole and skew quadrupole correctors that are required for storage ring operation. Additional development of the algorithm is planned, including control room tests on the existing APS storage ring.

\section{ACKNOWLEDGMENTS}

The author would like to thank M. Borland, J. Carwardine, G. Decker, L. Emery, Y. Sun, A. Xiao, A. Zholents for valuable discussions and help, and M. Borland for help with editing the manuscript. The work is supported by the U.S. Department of Energy, Office of Science, Office of Basic Energy Sciences, under Contract No. DEAC02-06CH11357.

[1] John N. Galayda, The Advanced Photon Source, in Proceedings of the Particle Accelerator Conference, Dallas, TX, 1995 (IEEE, New York, 1995), pp. 4-8.

[2] L. Farvacque et al., A low-emittance lattice for the ESRF, in Proceedings of the 4th International Particle Accelerator Conference, IPAC-2013, Shanghai, China, 2013 (JACoW, Shanghai, China, 2013), p. 79.

[3] M. Borland et al., Hybrid seven-bend-achromat lattice for the Advanced Photon Source Upgrade, Proceedings of 2015 IPAC (JACoW, Geneva, Switzerland, 2015), pp. 1776-1779, TUPJE063, http://accelconf.web.cern.ch/ AccelConf/IPAC2015/papers/tupje063.pdf.

[4] M. Borland, Survey of commissioning of recent storage ring light sources, Proceedings of IPAC 2015 (JACoW, Geneva, Switzerland, 2015), pp. 2482-2484, WEBD2, http://accelconf.web.cern.ch/AccelConf/IPAC2015/papers/ webd2.pdf.

[5] M. Aiba, R. Calaga, A. Morita, R.Tomas, and G. Vanbavinckhove, Optics correction in the LHC, in Proceedings of the 11th European Particle Accelerator Conference, Genoa, 2008 (EPS-AG, Genoa, Italy, 2008), WEPP025.

[6] G. Vanbavinckhove, M. Aiba, R. Calaga, and R.Tomas, Software package for optics measurement and correction in the LHC, in Proceedings of the International Particle Accelerator Conference, Kyoto, Japan (ICR, Kyoto, 2010), THPE026.

[7] R. Tomas et al., First beta-beating measurement in the LHC, in Proceedings of the 23rd Particle Accelerator Conference, Vancouver, Canada, 2009 (IEEE, Piscataway, NJ, 2009), WE6PFP021.

[8] T. Thomas et al., LHC optics model, measurements, and corrections, in Proceedings of the International Particle Accelerator Conference, Kyoto, Japan (ICR, Kyoto, 2010), TUXMH02.

[9] F. Willeke, Commissioning of NSLS-II, Proceedings of IPAC 2015 (JACoW, Geneva, Switzerland, 2015), pp. 11-16, http://accelconf.web.cern.ch/AccelConf/IPAC2015 /papers/moygb3.pdf, MOYGB3.

[10] G. Shen, M. Kraimer, P. Chu, and J. Wu, Design of an accelerator online simulator server using structured data, in Proceedings of the International Particle Accelerator Conference, Kyoto, Japan (ICR, Kyoto, 2010), WEPEB024.

[11] Swiss Light Source-2 Conceptual Design Report, http:// www.lib4ri.ch/archive/nebis/PSI_Berichte_000478272/ PSI-Bericht_17-03.pdf.

[12] J. Ousterhout, Tcl and the Tk Toolkit (Addison-Wesley, Reading, MA, 1994).

[13] M. Borland and L. Emery, The self-describing data sets file protocol and program toolkit, Proceedings of ICALEPCS 1995, Chicago, Illinois (1996), pp. 653-662, http://wwwbd.fnal.gov/icalepcs/abstracts/PDF/wpo43.pdf.

[14] R. Soliday, M. Borland, L. Emery, and H. Shang, New features in the SDDS toolkit, in Proceedings of the 20th Particle Accelerator Conference, PAC-2003, Portland, OR, 2003 (IEEE, New York, 2003), p. 3473.

[15] M. Borland, elegant: A flexible SDDS-compliant code for accelerator simulation, Report No. ANL/APS LS-287, 2000.

[16] M. Abliz et al., Septum magnet design for APS-U, Proceedings of NA-PAC 2016 (JACoW, Geneva, Switzerland, 2016), THPOA63, http://accelconf.web.cern .ch/AccelConf/napac2016/papers/thpoa63.pdf.

[17] A. Xiao, M. Borland, and C.-Y. Yao, On-axis injection scheme for ultra-low-emittance light sources, in Proceedings of the 25th Particle Accelerator Conference, PAC2013, Pasadena, CA, 2013 (IEEE, New York, 2013), WEPSM13.

[18] L. Emery, Application of model-independent analysis using the SDDS toolkit, in Proceedings of the 20th Particle Accelerator Conference, PAC-2003, Portland, OR, 2003 (IEEE, New York, 2003), p. 3464.

[19] L. Emery (private communication).

[20] J. Laskar, Secular evolution of the solar system over 10 million years, Astron. Astrophys. 198, 341 (1988).

[21] P. Castro et al., Betatron function measurement at LEP using the BOM 1000 turns facility, in Proceedings of the 15th Particle Accelerator Conference, PAC-1993, Washington, DC, 1993 (IEEE, New York, 1993), p. 2103 .

[22] J. Safranek, Experimental determination of storage ring optics using orbit response measurements, Nucl. Instrum. Methods Phys. Res., Sect. A 388, 27 (1997).

[23] V. Sajaev and L. Emery, Determination and correction of the linear lattice of the APS storage ring, in Proceedings of the 8th European Particle Accelerator Conference, Paris, 2002 (EPS-IGA and CERN, Geneva, 2002), pp. 742-744.

[24] A. Xiao and V. Sajaev, Simulation study of injection performance for the Advanced Photon Source Upgrade, Proceedings of IPAC 2015, Richmond, VA, USA (JACoW, Geneva, Switzerland, 2015), TUPJE075, http://accelconf .web.cern.ch/AccelConf/IPAC2015/papers/tupje075.pdf.

[25] Y. Sun (private communication).

[26] R. Lindberg (private communication). 